OPEN ACCESS

Edited by:

Nino Russo,

University of Calabria, Italy

Reviewed by:

Muhammad Jamil,

Riphah International University

Pakistan

Sk. Md. Abu Nayeem,

Aliah University, India

${ }^{*}$ Correspondence:

Farkhanda Afzal

farkhanda@mcs.edu.pk

Specialty section:

This article was submitted to

Theoretical and Computational

Chemistry,

a section of the journal

Frontiers in Chemistry

Received: 05 October 2020 Accepted: 09 December 2020 Published: 03 February 2021

Citation:

Shin DY, Hussain S, Afzal F, Park C Afzal D and Farahani MR (2021) Closed Formulas for Some New Degree Based Topological Descriptors Using M-polynomial and Boron Triangular Nanotube.

Front. Chem. 8:613873.

doi: 10.3389/fchem.2020.613873

\section{Closed Formulas for Some New Degree Based Topological Descriptors Using M-polynomial and Boron Triangular Nanotube}

\author{
Dong Yun Shin ${ }^{1}$, Sabir Hussain ${ }^{2}$, Farkhanda Afzal ${ }^{3 *}$, Choonkil Park ${ }^{4}$, Deeba Afzal ${ }^{2}$ and \\ Mohammad R. Farahani ${ }^{5}$ \\ ${ }^{1}$ Department of Mathematics, University of Seoul, Seoul, South Korea, ${ }^{2}$ Department of Mathematics and Statistics, The \\ University of Lahore, Lahore, Pakistan, ${ }^{3}$ Department of Humanities and Basic Sciences, Military College of Signals, National \\ University of Science and Technology, Islamabad, Pakistan, ${ }^{4}$ Research Institute for Natural Sciences, Hanyang University, \\ Seoul, South Korea, ${ }^{5}$ Department of Mathematics, Iran University of Science and Technology, Tehran, Iran
}

In this article, we provide new formulas to compute the reduced reciprocal randić index, Arithmetic geometric ${ }_{1}$ index, $S K$ index, $S K_{1}$ index, $S K_{2}$ index, edge version of the first zagreb index, sum connectivity index, general sum connectivity index, and the forgotten index using the M-polynomial and finding these topological indices for a boron triangular nanotube. We also elaborate the results with graphical representations.

Keywords: graph, chemical graph theory, M-polynomial, boron triangular nanotube, topological indices

\section{INTRODUCTION}

A chemical molecular structure is composed of atoms that join together with chemical bonds. This molecular structure is responsible for the chemical, physical, and biological properties of the chemical compound. A chemical graph theory is an important field of science, in which we study the formation and behavior of a chemical structure with the help of graph theory tools.

In graph theory a set of points is referred as graph $G$. These points are known as vertices. An edge is a line joining the two vertices. The number of edges that coincide at a vertex is considered to be the degree of the vertex and is represented as $d_{j}$ and the degree of the edge is defined as $d_{j k}=d_{j}+d_{k}-2 . E(G)$ represents the set of edges and $V(G)$ shows the set of vertices. In the chemical graph, an atom of the molecule is represented with the vertex of the graph, and the bond is considered as an edge.

There are many uses of chemical graph theory in different subjects, such as quantum chemistry, computer sciences, biology, stereochemistry, and engineering, which is explained by Gutman and Trinajstić (1972), Balaban (1985), Trinajstiéc (1992), Shirinivas et al. (2010), and Vergniory et al. (2017). With the help of chemical graph theory techniques, we convert a chemical molecule into a real number, which is referred to as a topological index and the molecular structure is examined through the topological indices. In this study, we try to develop mathematical methods for the calculation of topological indices. With the help of topological indices, Hosamani et al. (2017) studied the different physical properties like the molar volume, boiling points, and molar refraction, of the molecular structure. Rouvray (1986) and Ramakrishnan et al. (2013) describe the biological behavior, such as nutritive, stimulation of cell growth, toxicity, and $\mathrm{pH}$ regulation, of the chemical species, which are also characterized through topological indices. 
These topological indices are widely used in chemical graph theory to explain the different properties of the chemical structure. The carbon-hydrogen bond is not considered during the computation of topological indices because this bond does not have a serious effect on the properties of the chemical compound. During the QSAR and QSPR analysis, topological indices are widely used.

Gutman et al. (2014) formulated a reduced reciprocal randić index defined as $R R R=\sum_{j k \in E(G)} \sqrt{\left(d_{j}-1\right)\left(d_{k}-1\right)}$. Shigehalli and Kanabur (2015) presented the arithmetic geometric $c_{1}$ index defined as $A G_{1}=\sum_{j k \in E(G)} \frac{d_{j}+d_{k}}{2 \sqrt{d_{j} \cdot d_{k}}}$. Shigehalli and Kanabur (2016) also introduced the new indices defined as $S K$ index $=\sum_{j k \in E(G)} \frac{d_{j}+d_{k}}{2}, S K_{1}$ index $=\sum_{j k \in E(G)} \frac{d_{j} \cdot d_{k}}{2}$, and $S K_{2}$ index $=\sum_{j k \in E(G)}\left(\frac{d_{j}+d_{k}}{2}\right)^{2}$. Miličević et al. (2004) presented the first Zagreb index in term of edge degree defined as $E M_{1}=$ $\sum_{j k \in E(G)}\left(d_{j k}\right)^{2}$. Du et al. (2011) formulated the general sumconnectivity index defined as SCI $=\sum_{j k \in E(G)} \frac{1}{\sqrt{d_{j}+d_{k}}}$ and $S C I_{\lambda}=\sum_{j k \in E(G)}\left(d_{j}+d_{k}\right)^{\lambda}$. Gutman and Furtula (2015) presented the forgotten index represented with $F$ and defined as $F=\sum_{j k \in E(G)}\left(d_{j}^{2}+d_{k}^{2}\right)$.

\section{BORON TRIANGULAR NANOTUBE}

The analysis of a chemical molecular structure smaller than $100 \mathrm{~nm}$ is known as nanotechnology. Nanomaterials have many applications in different fields of nanoscience. The boron triangular nanotube $B T n t_{l q}$ is a well-known structure in nanomaterials with a wide range of applications in medicine, electronics, and computers as discussed by Menuel (2010), Bezugly et al. (2011), and Liu et al. (2018). With the help of this, experts are expected to revolutionize the world. The formation of the boron triangular nanotube is formed by a 2-D boron triangular nanosheet consisting of $l$ rows and $q$ columns. The first and the last column of the 2-D boron triangular nanosheet are connected to form a boron triangular nanotube.

A detailed analysis of Figures 1, 2 shows that there are only two types of vertex present in $B T n t_{l q}$. One which has a degree of four and the other has a degree of six. Now count the edges with smaller dimension and then by generalization and we obtain Table 1.

\section{M-POLYNOMIAL}

An algebraic polynomial has the ability to elaborate the chemical molecular structure. M-polynomial is such a polynomial that represents the graph. Deutsch and Klawzar (2015) formulated the M-polynomial and defined as $M(G, x, y)=$

$\sum_{\delta \leq u \leq v \leq \Delta} m_{u v}(G) x^{u} y^{v}$. Where $\delta$ represents the minimum degree of the vertex belonging to the vertex set $V(G), \Delta$ represents the maximum degree of the vertex belonging to the vertex set $V(G)$ and $m_{v v}(G)$ is the total number of edges $j k \in E(G)$ such that $\left\{d_{j}, d_{k}\right\}=\{u, v\}$.

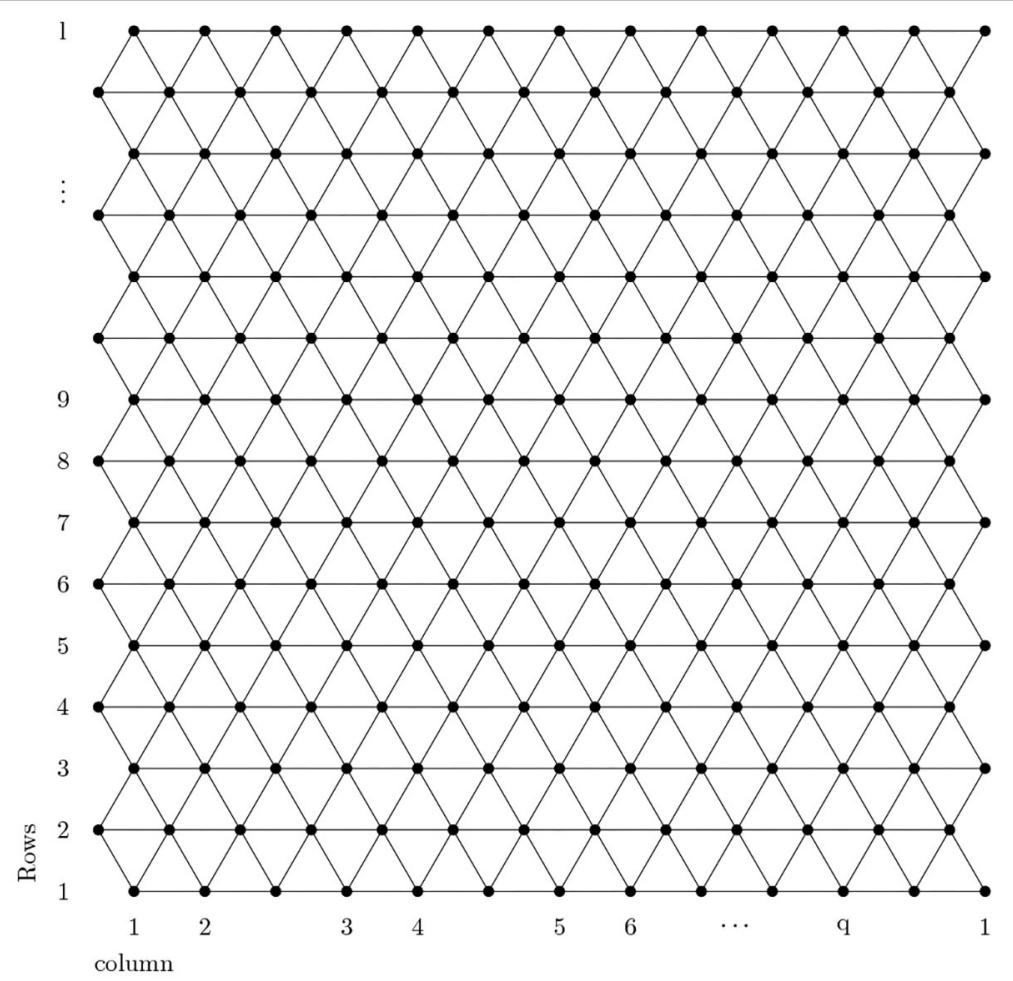

FIGURE 1 | Boron triangular nanosheet. 


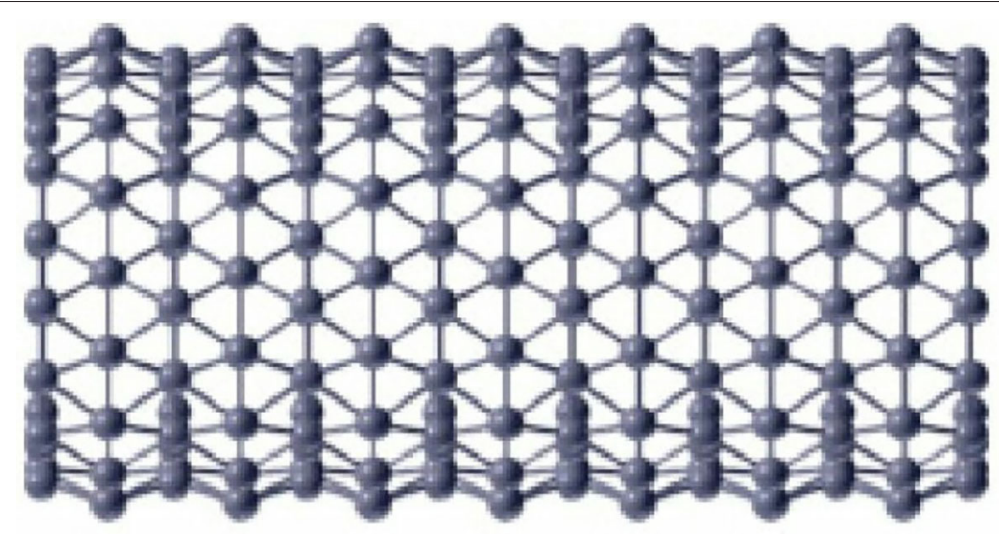

FIGURE 2 | Boron triangular nanotube (BTnt/q).

TABLE 1 | Edge partition of boron triangular nanotube $\left(B T n t_{/ q}\right)$.

\begin{tabular}{ll}
\hline$\left(\boldsymbol{d}_{u}, \boldsymbol{d}_{v}\right)$ & Number of edges \\
\hline$(4,4)$ & $3 q$ \\
$(4,6)$ & $6 q$ \\
$(6,6)$ & $\frac{q}{2}(9 /-24)$ \\
\hline
\end{tabular}

Deutsch and Klawzar (2015) introduced M-polynomial and in the same article nine topological indices closed formulas are given via m-polynomial. Some indices are calculated by Munir et al. (2016). In the past, Kang et al. (2018), Afzal et al. (2020a,b), Cancan et al. (2020a,b), and Khalaf et al. (2020) used these formulas to compute topological indices via $\mathrm{M}$ polynomial and a lot of work has been done in this area. One more set of nine topological indices has been computed by Afzal et al. (2020c). The F-index is also computed by Mondal et al. (2020). In this article, we formulated a new set of nine topological indices shown in Table 2. These formulas are used to compute the topological indices via M-polynomial for the chemical structure. This formulation is a useful achievement in the field the topological indices and opens a new research field. We apply the newly formulated indices on $B T n t_{l q}$.

\section{M-POLYNOMIAL OF BORON TRIANGULAR NANOTUBE}

Theorem 4.1. Let BTnt $t_{l q}$ be a Boron triangular nanotube where lq is the dimension of the BTnt $t_{q}$ then M-polynomial of BTnt $t_{q}$ is

$$
M\left[B \operatorname{Tnt}_{l q} ; x, y\right]=3 q x^{4} y^{4}+6 q x^{4} y^{6}+\frac{q}{2}(9 l-24) x^{6} y^{6}
$$

Proof: Let $B \operatorname{Tnt}_{l q}$ be a boron triangular nanotube then by using Figures 1, 2 and Table 1 the edge partition of boron triangular nanotube is consisting of three type of sets. The first edge partition represented with $E_{1}$ contains $3 q$ edges, in which $d_{j}$ and $d_{k}$ have the same value equal to 4 . The second edge partition
TABLE 2 | Topological indices derive from $M\left(B T n t_{l q} ; x, y\right)$.

\begin{tabular}{|c|c|c|}
\hline Topological index & & Derivation from $M\left(B T_{n} t_{l q} ; x, y\right)=g(x, y)$ \\
\hline$R R R\left[B \operatorname{Bnt}_{l q}\right]$ & $=$ & $D_{x}^{\frac{1}{2}} D_{y}^{\frac{1}{2}} Q_{y(-1)} Q_{x(-1)}[g(x, y)]_{x=y=1}$ \\
\hline$A G_{1}\left[B T n t_{l q}\right]$ & $=$ & $\frac{1}{2} D_{x} J S_{x}^{\frac{1}{2}} S_{y}^{\frac{1}{2}}[g(x, y)]_{x=1}$ \\
\hline$S K\left[B T n t_{/ q}\right]$ & $=$ & $\frac{1}{2}\left(D_{x}+D_{y}\right)[g(x, y)]_{x=y=1}$ \\
\hline $\mathrm{SK}_{1}\left[B \operatorname{Bnt}_{/ q}\right]$ & $=$ & $\frac{1}{2} D_{x} D_{y}[g(x, y)]_{x=y=1}$ \\
\hline$S k_{2}\left[B T n t_{l q}\right]$ & $=$ & $\frac{1}{4} D_{x}^{2} J[g(x, y)]_{x=y=1}$ \\
\hline$E M_{1}\left[B T n t_{/ q}\right]$ & $=$ & $D_{x}^{2} Q_{x(-2)} J[g(x, y)]_{x=1}$ \\
\hline$S C l\left[B T n t_{l q}\right]$ & $=$ & $S_{x}^{\frac{1}{2}} J[g(x, y)]_{x=1}$ \\
\hline$S C l_{\lambda}\left[B T n t_{/ q}\right]$ & $=$ & $D_{x}^{\lambda} J[g(x, y)]_{x=1}$ \\
\hline$F\left[B \operatorname{Tnt}_{/ q}\right]$ & $=$ & $\left(D_{x}^{2}+D_{y}^{2}\right)[g(x, y)]_{x=1}$ \\
\hline
\end{tabular}

Where the operator used are defined as

$D_{x} g(x, y)=x \frac{\partial(g(x, y))}{\partial x}, \quad D_{y} g(x, y)=y \frac{\partial(g(x, y))}{\partial y}, \quad J g(x, y)=g(x, x), \quad Q_{x(\alpha)} g(x, y)=x^{\alpha} g(x, y)$,

$$
\begin{array}{ll}
D_{x}^{\frac{1}{2}} g(x, y)=\sqrt{x \frac{\partial(g(x, y))}{\partial x}} \cdot \sqrt{g(x, y),} & D_{y}^{\frac{1}{2}} g(x, y)=\sqrt{y \frac{\partial(g(x, y))}{\partial y}} \cdot \sqrt{g(x, y),} \\
S_{x}^{\frac{1}{2}} g(x, y)=\sqrt{\int_{0}^{x} \frac{g(t, y)}{t} d t} \cdot \sqrt{g(x, y)}, & S_{y}^{\frac{1}{2}} g(x, y)=\sqrt{\int_{0}^{y} \frac{g(x, t)}{t} d t} \cdot \sqrt{g(x, y)}
\end{array}
$$

referred to as $E_{2}$ consists of $6 q$ edges $j k$, in which the value of $d_{j}$ is 4 and value of $d_{k}$ is 6 . The third edge partition named as $E_{3}$ contains $\frac{q}{2}(9 l-24)$ edges $j k$, in which $d_{j}=d_{k}=6$. Now, by using the definition of M-polynomial, we have

$$
\begin{aligned}
M\left(B \operatorname{Bin} t_{l q} ; x, y\right)= & \sum_{\delta \leq u \leq v \leq \Delta} m_{u v}\left(B_{\alpha} N T_{m n}\right) x^{u} y^{v} \\
= & \sum_{4 \leq u \leq v \leq 6} m_{u v}\left(B \operatorname{Tn} t_{l q}\right) x^{u} y^{v} \\
M\left(B \operatorname{BTn} t_{l q} ; x, y\right)= & \sum_{4 \leq 4} m_{44}\left(B \operatorname{Tn} t_{l q}\right) x^{4} y^{4}+\sum_{4 \leq 6} m_{46}\left(B \operatorname{Tn} t_{l q}\right) x^{4} y^{6} \\
& +\sum_{6 \leq 6} m_{66}\left(B \operatorname{Tn} t_{l q}\right) x^{6} y^{6} \\
M\left(B \operatorname{BTn} t_{l q} ; x, y\right)= & \left|E_{4,4}\right| x^{4} y^{4}+\left|E_{4,6}\right| x^{4} y^{6}+\left|E_{6,6}\right| x^{6} y^{6} \\
M\left[B \operatorname{BTn} t_{l q} ; x, y\right]= & 3 q x^{4} y^{4}+6 q x^{4} y^{6}+\frac{q}{2}(9 l-24) x^{6} y^{6} .
\end{aligned}
$$

The plot of $M\left[B \operatorname{Bnt}_{l q} ; x, y\right]$ is shown in Figure 3. 


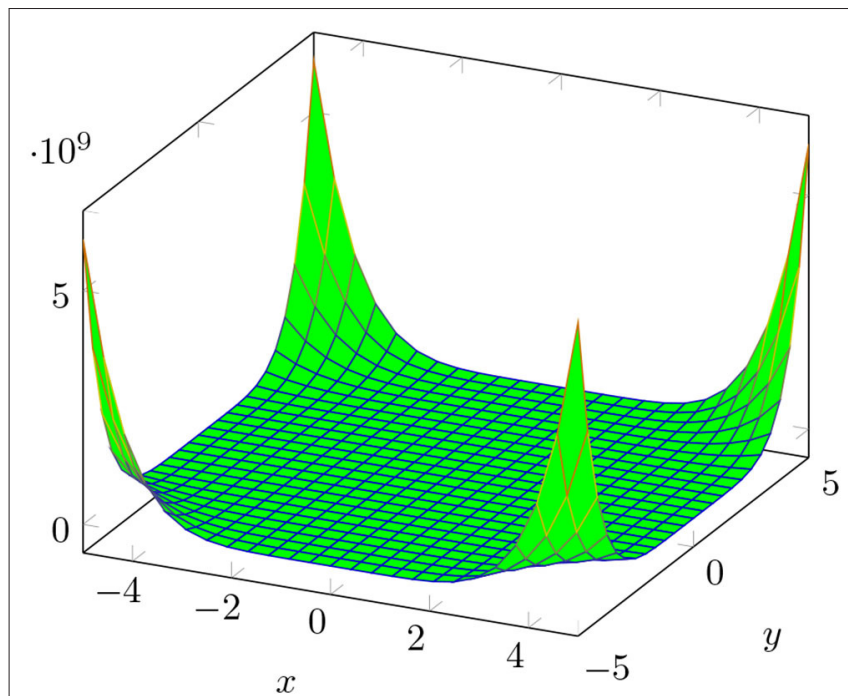

FIGURE 3 | 3D plots of M-polynomial of boron triangular nanotube BTnt $/$ for $l=q=4$.

\section{TOPOLOGICAL INDICES OF BORON TRIANGULAR NANOTUBE}

Theorem 5.1. Let BTnt $t_{l q}$ be a boron triangular nanotube and

$$
M\left[B \operatorname{Tnt}_{l q} ; x, y\right]=3 q x^{4} y^{4}+6 q x^{4} y^{6}+\frac{q}{2}(9 l-24) x^{6} y^{6},
$$

then

1. $R R R\left[B \operatorname{Tnt}_{l q}\right]=\frac{45}{2} q l+(6 \sqrt{15}-51) q$,

2. $A G_{1}\left[B \operatorname{Bnt}_{l q}\right]=\frac{9}{2} q l+\left(\frac{5 \sqrt{6}}{2}-9\right) q$,

3. $S K\left[B T n t_{l q}\right]=27 q l-30 q$,

4. $S K_{1}\left[B \operatorname{Brt} t_{l q}\right]=81 q l-120 q$,

5. $S K_{2}\left[B \operatorname{BTt} t_{l q}\right]=162 q l-234 q$,

6. $\operatorname{EM}_{1}\left[B \operatorname{Brt}_{l q}\right]=450 q l-708 q$,

7. $S C I\left[B \operatorname{Bnt}_{l q}\right]=\frac{3 \sqrt{3}}{4} q l+\left(\frac{3 \sqrt{2}}{4}+\frac{3 \sqrt{10}}{5}-2 \sqrt{3}\right) q$,

8. $\operatorname{SCI}_{\lambda}\left[B \operatorname{Brnt}_{l q}\right]=\frac{9}{2}(12)^{\lambda} q l+\left\{3(8)^{\lambda}+6(10)^{\lambda}-24(12)^{\lambda}\right\} q$,

9. $F\left[B \operatorname{Bnt}_{l q}\right]=324 q l-456 q$.

Proof: Let $\mathrm{M}\left[\mathrm{BTnt}_{l q} ; \mathrm{x}, \mathrm{y}\right]=\mathrm{g}(\mathrm{x}, \mathrm{y})$

$$
\begin{aligned}
Q_{x(-1)} g(x, y)= & 3 q x^{3} y^{4}+6 q x^{3} y^{6}+\frac{q}{2}(9 l-24) x^{5} y^{6}, \\
Q_{y(-1)} Q_{x(-1)} g(x, y)= & 3 q x^{3} y^{3}+6 q x^{3} y^{5}+\frac{q}{2}(9 l-24) x^{5} y^{5}, \\
D_{y}^{\frac{1}{2}} Q_{y(-1)} Q_{x(-1)} g(x, y)= & 3 \sqrt{3} q x^{3} y^{3}+6 \sqrt{5} q x^{3} y^{5} \\
& +\frac{\sqrt{5}}{2} q(9 l-24) x^{5} y^{5},
\end{aligned}
$$

$D_{x}^{\frac{1}{2}} D_{y}^{\frac{1}{2}} Q_{y(-1)} Q_{x(-1)} g(x, y)=9 q x^{3} y^{3}+6 \sqrt{15} q x^{3} y^{5}$

$$
+\frac{5}{2} q(9 l-24) x^{5} y^{5}
$$

$$
\begin{aligned}
& S_{y}^{\frac{1}{2}} g(x, y)=\frac{3}{2} q x^{4} y^{4}+\sqrt{6} q x^{4} y^{6}+\frac{q}{2 \sqrt{6}}(9 l-24) x^{6} y^{6}, \\
& S_{x}^{\frac{1}{2}} S_{y}^{\frac{1}{2}} g(x, y)=\frac{3}{4} q x^{4} y^{4}+\frac{\sqrt{6}}{2} q x^{4} y^{6}+\frac{q}{12}(9 l-24) x^{6} y^{6} \text {, } \\
& J S_{x}^{\frac{1}{2}} S_{y}^{\frac{1}{2}} g(x, y)=\frac{3}{4} q x^{8}+\frac{\sqrt{6}}{2} q x^{10}+\frac{q}{12}(9 l-24) x^{12}, \\
& D_{x} J S_{x}^{\frac{1}{2}} S_{y}^{\frac{1}{2}} g(x, y)=6 q x^{8}+5 \sqrt{6} q x^{10}+q(9 l-24) x^{12}, \\
& \frac{1}{2} D_{x} J S_{x}^{\frac{1}{2}} S_{y}^{\frac{1}{2}} g(x, y)=3 q x^{8}+\frac{5 \sqrt{6}}{2} q x^{10}+\frac{q}{2}(9 l-24) x^{12} \text {, } \\
& D_{x} g(x, y)=12 q x^{4} y^{4}+24 q x^{4} y^{6}+3 q(9 l-24) x^{6} y^{6}, \\
& D_{y} g(x, y)=12 q x^{4} y^{4}+36 q x^{4} y^{6}+3 q(9 l-24) x^{6} y^{6} \text {, } \\
& \left(D_{x}+D_{y}\right) g(x, y)=24 q x^{4} y^{4}+60 q x^{4} y^{6}+6 q(9 l-24) x^{6} y^{6} \text {, } \\
& \frac{1}{2}\left(D_{x}+D_{y}\right) g(x, y)=12 q x^{4} y^{4}+30 q x^{4} y^{6}+3 q(9 l-24) x^{6} y^{6} \text {, } \\
& D_{x} D_{y} g(x, y)=48 q x^{4} y^{4}+144 q x^{4} y^{6}+18 q(9 l-24) x^{6} y^{6}, \\
& \frac{1}{2}\left(D_{x} D_{y}\right) g(x, y)=24 q x^{4} y^{4}+72 q x^{4} y^{6}+9 q(9 l-24) x^{6} y^{6} \text {, } \\
& J g(x, y)=3 q x^{8}+6 q x^{10}+\frac{q}{2}(9 l-24) x^{12}, \\
& D_{x}^{2} J g(x, y)=192 q x^{8}+600 q x^{10}+72(9 l-24) x^{12}, \\
& \frac{1}{4} D_{x}^{2} \operatorname{Jg}(x, y)=48 q x^{8}+150 q x^{10}+18(9 l-24) x^{12}, \\
& Q_{x(-2)} J g(x, y)=3 q x^{6}+6 q x^{8}+\frac{q}{2}(9 l-24) x^{10}, \\
& D_{x}^{2} Q_{x(-2)} J g(x, y)=108 q x^{6}+384 q x^{8}+50 q(9 l-24) x^{10}, \\
& S_{x}^{\frac{1}{2}} J g(x, y)=\frac{3}{2 \sqrt{2}} q x^{8}+\frac{6}{\sqrt{10}} q x^{10}+\frac{q}{4 \sqrt{3}}(9 l-24) x^{12}, \\
& D_{x}^{\lambda} \operatorname{Jg}(x, y)=3(8)^{\lambda} q x^{8}+6(10)^{\lambda} q x^{10} \\
& +\frac{q}{2}(12)^{\lambda}(9 l-24) x^{12} \text {, } \\
& D_{x}^{2} g(x, y)=48 q x^{4} y^{4}+96 q x^{4} y^{6}+18 q(9 l-24) x^{6} y^{6} \text {, } \\
& D_{y}^{2} g(x, y)=48 q x^{4} y^{4}+216 q x^{4} y^{6}+18 q(9 l-24) x^{6} y^{6} \text {, } \\
& \left(D_{x}^{2}+D_{y}^{2}\right) g(x, y)=96 q x^{4} y^{4}+312 q x^{4} y^{6}+36 q(9 l-24) x^{6} y^{6} \text {. }
\end{aligned}
$$

1. $R R R\left[B \operatorname{Tnt}_{l q}\right]=D_{x}^{\frac{1}{2}} D_{y}^{\frac{1}{2}} Q_{y(-1)} Q_{x(-1)}[g(x, y)]_{x=y=1}$, $R R R\left[B \operatorname{Bnt}_{l q}\right]=\frac{45}{2} q l+(6 \sqrt{15}-51) q$.

2. $A G_{1}\left[B \operatorname{Bnt}_{l q}\right]=\frac{1}{2} D_{x} J S_{x}^{\frac{1}{2}} S_{y}^{\frac{1}{2}}[g(x, y)]_{x=1}$, $A G_{1}\left[B \operatorname{Bnt}_{l q}\right]=\frac{9}{2} q l+\left(\frac{5 \sqrt{6}}{2}-9\right) q$.

3. $S K\left[B \operatorname{Bnt} t_{l q}\right]=\frac{1}{2}\left(D_{x}+D_{y}\right)[g(x, y)]_{x=y=1}$, $S K\left[B \operatorname{Tnt}_{l q}\right]=27 q l-30 q$.

4. $S K_{1}\left[B T n t_{l q}\right]=\frac{1}{2} D_{x} D_{y}[g(x, y)]_{x=y=1}$, $S K_{1}\left[B \operatorname{Bnt}_{l q}\right]=81 q l-120 q$.

5. $S k_{2}\left[\right.$ BTnt $\left._{l q}\right]=\frac{1}{4} D_{x}^{2} J[g(x, y)]_{x=y=1}$, $S K_{2}\left[B \operatorname{Bint} t_{l q}\right]=162 q l-234 q$. 
$\mathbf{A}$

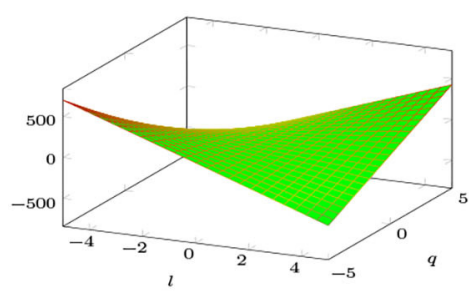

D

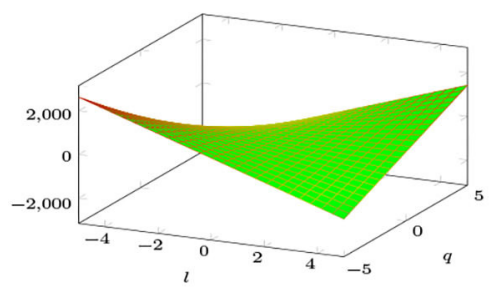

G

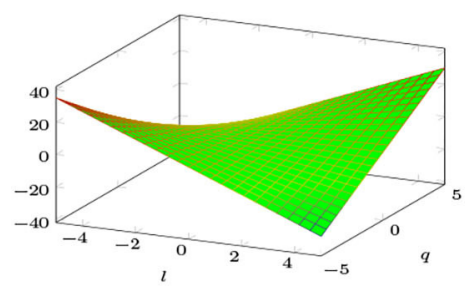

B



E

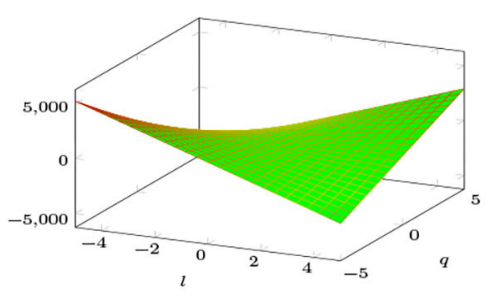

H

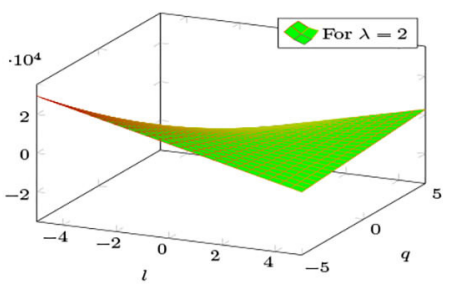

C

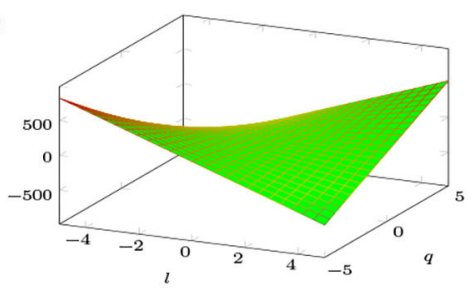

$\mathbf{F}$

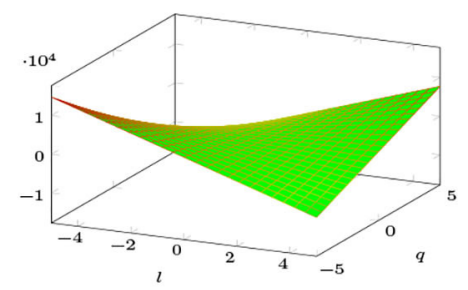

I

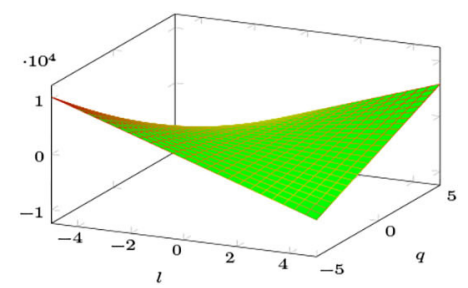

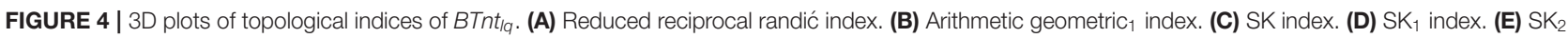
index. (F) Edge version of first Zagreb index. (G) Sum connectivity index. (H) General sum connectivity index. (I) Forgotten index.

6. $E M_{1}\left[B \operatorname{Brt}_{l q}\right]=D_{x}^{2} Q_{x(-2)} J[g(x, y)]_{x=1}$, $E M_{1}\left[B \operatorname{Bnt}_{l q}\right]=450 q l-708 q$.

7. $S C I\left[B \operatorname{Tnt}_{l q}\right]=S_{x}^{\frac{1}{2}} J[g(x, y)]_{x=1}$, $S C I\left[B \operatorname{Bnt}_{l q}\right]=\frac{3 \sqrt{3}}{4} q l+\left(\frac{3 \sqrt{2}}{4}+\frac{3 \sqrt{10}}{5}-2 \sqrt{3}\right) q$.

8. $S C I_{\lambda}\left[B \operatorname{Brt} t_{l q}\right]=D_{x}^{\lambda} J[g(x, y)]_{x=1}$, $S C I_{\lambda}\left[B \operatorname{Bnt}_{l q}\right]=\frac{9}{2} 12^{\lambda} q l+\left\{3(8)^{\lambda}+6(10)^{\lambda}-24(12)^{\lambda}\right\} q$.

9. $F\left[B \operatorname{Bin} t_{l q}\right]=\left(D_{x}^{2}+D_{y}^{2}\right)[g(x, y)]_{x=1}$, $F\left[B \operatorname{Bnt}_{l q}\right]=324 q l-456 q$.

Figure 4 shows a graphical analysis of topological indices of $B T n t_{l q}$. With the help of these graphs, we observe the behavior of the topological indices regarding the different parameters involved. These visualizations are shown to be identical but have different gradients.

\section{CONCLUSION}

The formulation of new formulas, placed in Table 2, to compute the topological indices for the molecular structure via $\mathrm{M}$ - polynomial lead to a new era in the computational field. In this research work, we compute $M\left(B \operatorname{Tnt}_{l q} ; x, y\right)$ and with the help of this polynomial, we find the various topological invariants given in Table 2. We also presented the graphical presentation of MPolynomial and topological indices. This visualization helps us to understand results against parameters.

\section{DATA AVAILABILITY STATEMENT}

The original contributions presented in the study are included in the article/supplementary material, further inquiries can be directed to the corresponding author/s.

\section{AUTHOR CONTRIBUTIONS}

DS: supervision, resources, and funding. $\mathrm{SH}$ : writing original draft, computations, and graphs. FA: editing, proof reading, and methodology. CP: investigation, validation, and funding. DA: conceptualization, supervision, and programming. MF: resources, proof reading, and validation. All authors contributed to the article and approved the submitted version. 


\section{REFERENCES}

Afzal, D., Hussain, S., Aldemir, M., Farahani, M., and Afzal, F. (2020a). New degree-based topological descriptors via m-polynomial of boron $\alpha$-nanotube. Eurasian Chem. Commun. 2, 1117-1125. doi: 10.22034/ecc.2020.254614.1092

Afzal, F., Hussain, S., Afzal, D., and Hameed, S. (2020b). M-polynomial and topological indices of zigzag edge coronoid fused by starphene. Open Chem. 18, 1362-1369. doi: 10.1515/chem-2020-0161

Afzal, F., Hussain, S., Afzal, D., and Razaq, S. (2020c). Some new degree based topological indices via M-polynomial. J. Inform. Optimiz. Sci. 41, 1061-1076. doi: 10.1080/02522667.2020.1744307

Balaban, A. T. (1985). Applications of graph theory in chemistry. J. Chem. Inform. Model. 25, 334-343. doi: 10.1021/ci00047a033

Bezugly, V., Kunstmann, J., Grundkötter-Stock, B., Frauenheim, T., Niehaus, T., and Cuniberti, G. (2011). Highly conductive boron nanotubes: transport properties, work functions, and structural stabilities. ACS Nano 5, 4997-5005. doi: $10.1021 / \mathrm{nn} 201099$ a

Cancan, M., Afzal, D., Hussain, S., Maqbool, A., and Afzal, F. (2020a). Some new topological indices of silicate network via M-polynomial. J. Discrete Math. Sci. Cryptogr. 23, 1157-1171. doi: 10.1080/09720529.2020.1809776

Cancan, M., Ediz, S., Mutee-Ur-Rehman, H., and Afzal, D. (2020b). M-polynomial and topological indices poly (EThyleneAmidoAmine) dendrimers. J. Inform. Optimiz. Sci. 41, 1117-1131. doi: 10.1080/02522667.2020.1745383

Deutsch, E., and Klawzar, S. (2015). M-polynomial and degree-based topological indices. Iran. J. Math. Chem. 6, 93-102.

Du, Z., Zhou, B., and Trinajstić, N. (2011). On the general sum-connectivity index of trees. Appl. Math. Lett. 24, 402-405. doi: 10.1016/j.aml.2010.10.038

Gutman, I., and Furtula, B. (2015). A forgotten topological index. J. Math. Chem. 53, 1184-1190. doi: 10.1007/s10910-015-0480-z

Gutman, I., Furtula, B., and Elphick, C. (2014). Three new/old vertex-degreebased topological indices. MATCH Commun. Math. Comput. Chem. 72, 617632.

Gutman, I., and Trinajstić, N. (1972). Graph theory and molecular orbitals. Total $\varphi$-electron energy of alternant hydrocarbons. Chem. Phys. Lett. 17, 535-538. doi: 10.1016/0009-2614(72)85099-1

Hosamani, S., Perigidad, D., Jamagoud, S., Maled, Y., and Gavade, S. (2017). QSPR analysis of certain degree based topological indices. J. Stat. Appl. Probabil. 6, 361-371. doi: 10.18576/jsap/060211

Kang, S. M., Nazeer, W., Gao, W., Afzal, D., and Gillani, S. N. (2018). Mpolynomials and topological indices of dominating David derived networks. Open Chem. 16, 201-213. doi: 10.1515/chem-2018-0023
Khalaf, A. J. M., Hussain, S., Afzal, D., Afzal, F., and Maqbool, A. (2020). M-polynomial and topological indices of book graph. J. Discrete Math. Sci. Cryptogr. 23, 1217-1237. doi: 10.1080/09720529.2020.1809115

Liu, J.-B., Shaker, H., Nadeem, I., and Hussain, M. (2018). Topological aspects of boron nanotubes. Adv. Mater. Sci. Eng. 12:134. doi: 10.1155/2018/5729291

Menuel, P. (2010). Computational aspects of carbon and boron nanotubes. Molecules 15, 8709-8722. doi: 10.3390/molecules 15128709

Miličević, A., Nikolić, S., and Trinajstić, N. (2004). On reformulated Zagreb indices. Mol. Divers 8, 393-399. doi: 10.1023/B:MODI.0000047504.14261.2a

Mondal, S., De, N., and Pal, A. (2020). Topological properties of networks using M-polynomial approach. Konuralp J. Math. 8, 97-105.

Munir, M., Nazeer, W., Rafique, S., and Kang, S. M. (2016). M-polynomial and related topological indices of nanostar dendrimets. Symmetry 8:97. doi: 10.3390/sym8090097

Ramakrishnan, S., Senbagamalar, J., and Babujee, J. B. (2013). Topological indices of molecular graphs under specific chemical reactions. Int. J. Comput. Algorithm 2, 68-74. doi: 10.20894/IJCOA.101.002.001.019

Rouvray, D. H. (1986). The prediction of biological activity using molecular connectivity indices. Acta Pharma. Jugoslavica 36, 239-252.

Shigehalli, V., and Kanabur, R. (2015). Arithmetic-geometric indices of path graph. J. Comp. Math. Sci. 6, 19-24.

Shigehalli, V., and Kanabur, R. (2016). Computing degree based topological indices of polyhex nanotube. J. Math. Nanosci. 6, 47-55. doi: 10.22061/JMNS.2016.525

Shirinivas, S. G., Vetrivel, S., and Elango, N. M. (2010). Applications of graph theory in computer science an overview. Int. J. Eng. Sci. Technol. 2, 4610-4621.

Trinajstić, N. (1992). Chemical Graph Theory. New York, NY: Routledge.

Vergniory, M. G., Elcoro, L., Wang, Z., Cano, J., Felser, C., Aroyo, M. I., et al. (2017). Graph theory data for topological quantum chemistry. Phys. Rev. E 96:023310. doi: 10.1103/PhysRevE.96.023310

Conflict of Interest: The authors declare that the research was conducted in the absence of any commercial or financial relationships that could be construed as a potential conflict of interest.

Copyright (c) 2021 Shin, Hussain, Afzal, Park, Afzal and Farahani. This is an openaccess article distributed under the terms of the Creative Commons Attribution License (CC BY). The use, distribution or reproduction in other forums is permitted, provided the original author(s) and the copyright owner(s) are credited and that the original publication in this journal is cited, in accordance with accepted academic practice. No use, distribution or reproduction is permitted which does not comply with these terms. 